\title{
Rhabdomyolysis During Laparoscopic Hepatic Subsegmentectomy
}

\author{
Pyeong Hoo Park, MD, Daelim Jee, MD \\ Department of Anesthesiology and Pain Medicine, Yeungnam University College of Medicine, Daegu, South Korea \\ (both authors).
}

\begin{abstract}
Introduction: Rhabdomyolysis is very rare in laparoscopic partial resection surgery of the liver. The muscles of the buttock, shoulder girdles, or back are usually injured with the patient in the supine position.

Case Description: We present a case showing peaked T waves and widened QRS complexes in electro-cardiograph that occurred 2 hours after the beginning of laparoscopic hepatic subsegmentectomy for hepatocellular carcinoma. Hyperkalemia and myoglobinemia quickly confirmed rhabdomyolysis during surgery, whereas postoperative creatine phosphokinase levels were normal. Postoperative evaluation suggested that the muscles of the right forearm were affected.

Discussion: The process of rhabdomyolysis appeared to begin early and unexpectedly in the muscles of the right forearm. An elevation of serum myoglobin levels without elevation of creatine phosphokinase levels suggests that serum myoglobin appears to have a crucial role in the diagnosis of early-phase rhabdomyolysis during surgery.
\end{abstract}

Key Words: Rhabdomyolysis, Laparoscopic surgery, Segmentectomy, Liver.

Citation Park PH, Jee D. Rhabdomyolysis during laparoscopic hepatic subsegmentectomy. CRSLS e2014.00190. DOI: 10.4293/CRSLS.2014.00190.

Copyright ( 2014 SLS This is an open-access article distributed under the terms of the Creative Commons Attribution-Noncommercial-ShareAlike 3.0 Unported license, which permits unrestricted noncommercial use, distribution, and reproduction in any medium, provided the original author and source are credited.

Address correspondence to: Daelim Jee, MD, Department of Anesthesiology and Pain Medicine, Yeungnam University College of Medicine, Daegu, Republic of Korea. Telephone: +82-53-620-3364, Fax: +82-53-626-5275, E-mail: adjee@med.yu.ac.kr.

\section{INTRODUCTION}

Rhabdomyolysis can occur in surgical patients with risk factors. A prolonged surgical time especially with patients in the lithotomy or lateral decubitus position, ${ }^{1,2}$ certain medications (such as statin, cyclosporin A, and colchicine), ${ }^{3}$ and concomitant diseases ${ }^{3-5}$ of patients are implicated in rhabdomyolysis. In liver transplantation cases, rhabdomyolysis has been reported in recipients. However, in cases of partial resection surgery of the liver, rhabdomyolysis is surprisingly rare. Our literature review identified only one case of rhabdomyolysis after partial hepatectomy. ${ }^{6}$

Rhabdomyolysis can give rise to acute metabolic acidosis, life-threatening hyperkalemia, myoglobinemia, and myoglobinuria. We present a case showing tall peaked $\mathrm{T}$ waves and widened QRS complexes on the patient's electrocardiographic (ECG) moniter during laparoscopic hepatic subsegmentectomy for hepatocellular carcinoma, which eventually led to the diagnosis of rhabdomyolysis.

\section{CASE REPORT}

A 54-year-old patient (height, $161 \mathrm{~cm}$; weight, $73 \mathrm{~kg}$ ) was scheduled for laparoscopic hepatic subsegmentectomy because of hepatocellular carcinoma. He had type 2 diabetes that had been well controlled, alcoholic liver cirrhosis with thrombocytopenia (platelet count, $73,000 / \mu \mathrm{L}$ ), and a history of hematemesis from variceal bleeding. His medications included glimepiride, $2 \mathrm{mg}$; metformin, 500 $\mathrm{mg}$; and voglibose, $0.3 \mathrm{mg}$; each twice daily. He denied other illnesses including neuromuscular system illnesses, and clinical abnormalities were not found on physical examination. Preoperative laboratory tests showed a hemoglobin level of $13.9 \mathrm{~g} / \mathrm{dL}$; serum sodium, potassium, and chloride levels of $138 \mathrm{mEq} / \mathrm{L}, 5.1 \mathrm{mEq} / \mathrm{L}$, and 102 $\mathrm{mEq} / \mathrm{L}$, respectively; and a serum creatinine level of 1.17 $\mathrm{mg} / \mathrm{dL}$. Preoperative chest radiography and echocardiography findings were unremarkable.

The patient fasted for 8 hours, and his medications were continued on the day of surgery. Preoperative vital signs in the operating room showed a blood pressure of 
$110 / 70 \mathrm{~mm} \mathrm{Hg}$ and heart rate of 60 beats/min. Glycopyrrolate, $0.2 \mathrm{mg}$, was injected for premedication before anesthesia. After noninvasive monitors were established (left arm noninvasive blood pressure, lead II on the electrocardiogram, end-tidal carbon dioxide, and bispectral index), remifentanil was infused at a rate of $0.15 \mu \mathrm{g} / \mathrm{kg} / \mathrm{min}$ and anesthesia was induced with 100 $\mathrm{mg}$ of propofol and $3 \mathrm{vol} \%$ of sevoflurane in 50\% $\mathrm{N}_{2} \mathrm{O} / \mathrm{O}_{2}$. Tracheal intubation was facilitated with $50 \mathrm{mg}$ of rocuronium. Positive pressure ventilation was delivered to maintain end-tidal carbon dioxide tension of 33 to $37 \mathrm{~mm} \mathrm{Hg}$. General anesthesia was maintained with a mixture of $\mathrm{N}_{2} \mathrm{O} / \mathrm{O}_{2}$, sevoflurane, and continuous infusion of remifentanil. A right subclavian central venous catheter and the left radial artery were cannulated, and a Foley urinary catheter was inserted, shortly after endotracheal intubation. The surgery was performed with the patient in the supine position with the upper extremities abducted $60^{\circ}$ on the arm boards. The patient's arms rested on the soft pads, and no external compression was applied to the arms during surgery.

Two hours after the start of the operation, the appearance of QRS complex widening and tall peaked $\mathrm{T}$ waves was noticed on the ECG monitor. Hyperkalemia was suspected, and the concentrations of serum potassium and ionized calcium were immediately measured (Table 1). After confirmation of hyperkalemia by a repeated measure of the potassium concentration, intravenous injection of $5 \mathrm{U}$ of regular insulin in $8 \mathrm{~g}$ of $20 \%$ dextrose solution and $300 \mathrm{mg}$ of $3 \%$ calcium chloride, followed by continuous infusion of $10 \mathrm{U}$ of regular insulin in 100 $\mathrm{mL}$ of $0.9 \%$ normal saline solution, was performed to control the life-threatening hyperkalemia. To investigate the causes of hyperkalemia, a blood sample was drawn to analyze arterial blood gas, lactate, myoglobin, and creatine phosphokinase (CPK) levels; mild myoglobinemia was found to be present (Table 1). The urine color appeared clear on visual inspection. Thirty minutes later, the serum potassium and ionized calcium levels measured $4.5 \mathrm{mEq} / \mathrm{L}$ and $1.09 \mathrm{mmol} / \mathrm{L}$, respectively, with a serum glucose concentration of $116 \mathrm{mg} /$ dL. ECG abnormalities returned to normal. Surgery was completed uneventfully. Intraoperative hemodynamics was well maintained with a systolic blood pressure $>90$ $\mathrm{mm} \mathrm{Hg}$ and heart rate of 60 to 70 beats $/ \mathrm{min}$. The surgical time was 250 minutes. We infused $3.6 \mathrm{~L}$ of intraoperative crystalloids and transfused $8 \mathrm{U}$ of platelet concentrates. The intraoperative urine output was 270 $\mathrm{mL}$. After completion of the operation, the patient's trachea was extubated, and he was transferred to the

\begin{tabular}{ll}
\hline \multicolumn{1}{c}{ Table 1. } \\
Laboratory Test Results After ECG Changes \\
\hline
\end{tabular}

intensive care unit. Postoperative analgesia was started with patient-controlled analgesia using fentanyl.

The muscles of the neck, shoulder girdles, back, and buttocks were inspected and palpated, and signs and symptoms suggestive of rhabdomyolysis were not found on the physical examination. The patient insisted that the motor function of the right upper extremity was preserved. Instead, he complained of a weak handgrip, as well as decreased sensation and paresthesia in the right forearm. No signs of swelling or color change were observed on the right forearm. Neurologic examination showed mild hypesthesia and paresthesia in the right hand and forearm. Handgrip strength with wrist flexion and extension was reduced to grade 4 on the Medical Research Council scale. Other muscles including the muscles of the hand, elbow, shoulder, and neck contracted normally against full resistance. Serum CPK levels measured on the first and second postoperative day were in the normal range (Table 1), and the patient's sensory and motor functions returned to normal on the second postoperative day. The postoperative course was uneventful, with stable vital signs and good urine output. He was discharged home without any sequelae on the fourteenth postoperative day. 


\section{DISCUSSION}

Our patient had a longstanding history of chronic alcoholism, liver cirrhosis, and diabetes, which all have been considered risk factors for rhabdomyolysis. ${ }^{3,4,7-9}$ In diabetic patients, the well-known vascular changes—such as microangiopathy, atheroma embolization, or vascular occlusion with consequent muscle edema, which reduces capillary blood flow-may contribute to muscle injury.? One case series reported that idiopathic rhabdomyolysis often occurs in patients with liver cirrhosis and suggested that defective muscle metabolism due to hepatic dysfunction results in rhabdomyolysis in these patients. ${ }^{8}$ Acute and chronic alcohol intoxication also has direct toxicity to muscle. ${ }^{3,7}$ It was reported that multiple causes were present in 60\% of patients assayed and multiple muscle insults are usually needed to produce rhabdomyolysis unless an underlying myopathy is present. ${ }^{10}$ In our opinion, rhabdomyolysis would not have occurred early if our patient had not had risk factors. These risk factors seem to play a significant role in the development of rhabdomyolysis. Our patient may have had vulnerable muscles from the long-lasting effects of these risk factors.

When one takes the multiple risk factors into consideration, the occurrence of rhabdomyolysis may not be a surprise. However, our patient is interesting regarding several points. First, he seemed to have the skeletal muscle injury in unexpected muscles. Rhabdomyolysis is rare in the supine position, and when it occurs in the supine position, the muscles of the buttocks, shoulder girdles, and back are usually involved because the skeletal muscle injury is usually subject to immobilization, compression pressure, and resultant ischemia during surgery. Our patient was devoid of symptoms, and physical examination showed no sign of injury in those muscles. Strangely, the patient presented with the symptoms of rhabdomyolysis, ${ }^{5}$ which are paresthesia, hypesthesia, and distal muscle weakness, such as decreased handgrip strength and decreased ability to perform wrist flexion and extension, in the right forearm muscles on the immediate postoperative evaluation. The forearm muscles are not the usual site of muscle injury in the supine position during surgery. Therefore, in our opinion, it is reasonable to assume that the muscles having symptoms (ie, the right forearm muscles) are more likely to be injured than the muscles having no symptoms, although we cannot exclude the possibility of asymptomatic rhabdomyolysis of the buttock or shoulder girdle or any other muscles. Second, the process of rhabdomyolysis occurred within 2 hours of the beginning of surgery, as indicated by the early ECG changes and subsequent identification of myoglobinemia. The early development of rhabdomyolysis is surprising because prolonged immobilization and ischemia longer than 4 hours usually cause hyperkalemia ${ }^{11}$ and rhabdomyolysis in the supine position. ${ }^{2,12}$ Third, the CPK levels were not elevated when measured postoperatively in our patient. It has been reported that CPK levels are normal or only moderately elevated in zidovudine-induced rhabdomyolysis. ${ }^{10,13}$ It has also been reported that the CPK levels were normal or mildly elevated in patients with diabetic muscle infarction. ${ }^{9}$ Moreover, the diagnosis can also be made by plasma measurement of myoglobin levels even without measurement of CPK levels, ${ }^{14}$ and some authors reported a case of rhabdomyolysis without describing the CPK levels. ${ }^{15}$ We could not find evidence in the literature that CPK levels are always elevated in rhabdomyolysis cases. These reports and our case suggest that normal CPK levels may not exclude rhabdomyolysis.

The diagnosis of rhabdomyolysis is usually made by CPK measurement because CPK is cleared slowly, with a peak at 1 to 3 days and a decline at 3 to 5 days after the cessation of muscle injury. ${ }^{3}$ However, the measurement of CPK levels may cause a problem in the diagnosis of rhabdomyolysis: because CPK levels rise more slowly than myoglobin levels do after muscle injury, ${ }^{16} \mathrm{CPK}$ levels may not reflect the early phase of rhabdomyolysis, as in our case during surgery. In contrast, serum myoglobin levels rise early with a short half-life (2-3 hours). ${ }^{3}$ Thus the measurement of serum myoglobin levels appears to have a crucial role in the detection of early-phase rhabdomyolysis during surgery. In addition, in the case of normal CPK levels, as described earlier, measurement of serum myoglobin levels appears to play a role in the diagnosis.

It is not known why rhabdomyolysis is reported more rarely in partial resection surgery of the liver than in liver transplantation surgery. Liver transplantation surgery is often a long operation, and consequently, patients undergoing it are obviously at risk for rhabdomyolysis. Furthermore, hemodynamic perturbations, which might impair blood flow to the muscles, are frequent, in addition to the risk factors that the transplantation patients may already have. The use of immune suppressants such as cyclosporin A may contribute to the development of rhabdomyolysis after transplantation surgery. In contrast, the duration of surgery is shorter, hemodynamics during surgery is less disturbed, and drugs such as cyclosporin A are not needed in patients undergoing partial resection surgery of the liver, although the patients may have the same risk factors. These factors may partly explain why rhab- 
domyolysis is rarely reported in partial resection surgery of the liver.

In conclusion, the process of rhabdomyolysis appeared to begin early and unexpectedly in the muscles of the right forearm. A mild elevation of serum myoglobin levels without elevation of CPK levels suggests that serum myoglobin appears to have a crucial role in the diagnosis of earlyphase rhabdomyolysis during surgery.

\section{References:}

1. Pfeffer SD, Halliwill JR, Warner MA. Effects of lithotomy position and external compression on lower leg muscle compartment pressure. Anesthesiology. 2001;95:632-636.

2. Targa L, Droghetti L, Caggese G, Zatelli R, Roccella P. Rhabdomyolysis and operating position. Anaesthesia. 1991;46:141143.

3. Huerta-Alardín AL, Varon J, Marik PE. Bench-to-bedside review: rhabdomyolysis-an overview for clinicians. Crit Care. 2005;9:158-169.

4. Woolley SL, Smith DR. Acute compartment syndrome secondary to diabetic muscle infarction: case report and literature review. Eur J Emerg Med. 2006;13:113-116.

5. Youssef T, Abd-Elaal I, Zakaria G, Hasheesh M. Bariatric surgery: rhabdomyolysis after open Roux-en-Y gastric bypass: a prospective study. Int J Surg. 2010;8:484-488.

6. Lee KW, Kim SH, Han SS, et al. Use of an upper midline incision for living donor partial hepatectomy: a series of 143 consecutive cases. Liver Transpl. 2011;17:969-975.
7. Preedy VR, Salisbury JR, Peters TJ. Alcoholic muscle disease: features and mechanisms. J Pathol. 1994;173:309-315.

8. Baek JE, Park DJ, Kim HJ, Lee JD, Chang SH. The clinical characteristics of rhabdomyolysis in patients with liver cirrhosis. J Clin Gastroenterol. 2007;41:317-321.

9. Silberstein L, Britton KE, Marsh FP, Raftery MJ, D'Cruz D. An unexpected cause of muscle pain in diabetes. Ann Rheum Dis. 2001;60:310-312.

10. Melli G, Chaudhry V, Cornblath DR. Rhabdomyolysis: an evaluation of 475 hospitalized patients. Medicine. 2005;84:377385 .

11. Kaye AD, Riopelle JM. Intravascular fluid and electrolyte physiology. In: Miller RD, ed. Miller's Anesthesia. Philadelphia, PA: Elsevier Churchill Livingstone; 2010:1705-1737.

12. Choufane S, Lemogne M, Jacob L. Unexpected rhabdomyolysis with myoglobinuria in a patient in the supine position. Eur J Anaesthesiol. 1998;15:493-496.

13. Sieb JP, Gillessen T. Iatrogenic and toxic myopathies. Muscle Nerve. 2003;27:142-156.

14. Lagandré S, Arnalsteen L, Vallet B, et al. Predictive factors for rhabdomyolysis after bariatric surgery. Obes Surg. 2006;16:13651370 .

15. Hung CM, Huang CC, Jeng LB, et al. Impaired renal function after liver transplantation: role of rhabdomyoglobinuria. Transplant Proc. 2000;32:2227-2228.

16. Khan FY. Rhabdomyolysis: a review of the literature. Neth J Med. 2009;67:272-283. 